\title{
Pilot study on the effectiveness of the conventional CROS, the transcranial CROS and the BAHA transcranial CROS in adults with unilateral inner ear deafness
}

\author{
Myrthe K. S. Hol • Sylvia J. W. Kunst • \\ Ad F. M. Snik · Cor W. R. J. Cremers
}

Received: 26 July 2009/ Accepted: 22 October 2009/Published online: 11 November 2009

(C) The Author(s) 2009. This article is published with open access at Springerlink.com

\begin{abstract}
The objective of the present pilot study is to evaluate the effectiveness of three conventional contralateral routing of sound (CROS) hearing aids in adults with unilateral inner ear deafness. The study included tertiary referral center. Ten patients with unilateral inner ear deafness and normal hearing in the contralateral ear were selected to evaluate three different methods of amplification: the CROS hearing aid, the completely in the canal hearing aid and the bone-anchored hearing aid CROS (BAHA). Each of the three hearing aids was tried in a random order for a period of 8 weeks. Audiometric performance, including speech-in-noise, directional hearing and subjective benefit were measured after each trial period, using the APHAB, SSQ and single-sided deafness questionnaire. Sound localization performance was essentially at chance level in all four conditions. Mixed results were seen on the other patient outcome measures that alternated in favor of one of the three CROS devices. After the trial, three patients chose to be fitted with the BAHA CROS and one with the conventional CROS. In conclusion, most of the patients experienced some degree of benefit with each of the three hearing aids. Preference for one of the three hearing aids was independent of the order in which they were tried. It would be worthwhile to formulate selection criteria; still, we recommend that all patients with unilateral inner ear deafness should be offered a trial with at least the BAHA CROS.
\end{abstract}

M. K. S. Hol ( $\square)$ · S. J. W. Kunst · A. F. M. Snik ·

C. W. R. J. Cremers

Department of Otorhinolaryngology, Head and Neck Surgery,

Radboud University Nijmegen Medical Centre,

9101, 6500 HB Nijmegen, The Netherlands

e-mail: m.hol@kno.umcn.nl
Keywords CROS devices - Unilateral inner ear deafness

\section{Introduction}

Before considering a hearing aid in patients with singlesided deafness (SSD), it is important to investigate their medical and social backgrounds. Harford and Dodds [1] were among the first who advocated contralateral routing of sound (CROS) amplification for such patients to eliminate head shadow. These authors found that the degree of success depended on the motivation of the patient and the listening demands imposed by their lifestyle and working environment. They applied what is nowadays known as the "conventional CROS" device. It comprises a microphone placed near the impaired ear and an amplifier (hearing aid) near the normal ear. The signal is presented to the normal ear via an open ear mould. Conventional CROS devices only transmit the frequencies of above $1,000 \mathrm{~Hz}$ through this open ear mould, which results in a "tinny" sound that might help the patient to localize sounds. Acceptance of this conventional CROS device by the patients was related to the level of hearing in their best ear. When hearing was within normal limits, the success rate was low: only one out of 12 patients accepted the CROS device. However, when mild high frequency hearing loss was present, the success rate was increased to $54 \%$ [1].

Lotterman and Kasten [2] studied the effect of a conventional CROS device when words were presented against a background of cafeteria noise. They observed favorable results when the speech was presented near the impaired ear, but unfavorable results when the speech was presented near the normal ear. Markides [3] reported similar results. $\mathrm{He}$ also tested directional hearing, but found that none of his patients could localize sounds. 
With the conventional CROS, sound is received on the impaired hearing side and transmitted by a wire around the neck, or by wireless FM transmission, to the best ear $[4,5]$. However, many patients find it unpleasant to have an ear mould in their best ear and a cord around their neck. The ear mould causes at least partial occlusion of the best ear, which is a key consideration; because the adaptive behavior learned by individuals with unilateral hearing loss often involves turning the best ear towards the sound source. Nowadays, the conventional CROS is available with wireless FM transmission; however, the wire around the neck is still used in the test setting as this is known to have little effect on the hearing results, but the FM link is more accepted from an esthetic point of view.

Another option is the use of bone conduction in the form of a "transcranial CROS" device. In 1960, Fowler [6] suggested the use of a bone conduction hearing aid (in a spectacle frame) near the deaf ear. This would stimulate the normal cochlea (cross stimulation) by bone conduction i.e. through the skull. No results of this application were presented at that time. Probably, the first, comprehensive study on transcranial CROS via bone conduction was published in 1991 by Welling et al. [7]. They used the implantable audiant bone conductor and reported positive results in some of their patients. One of the main limitations of their device was insufficient output [8].

In 2000, Vaneeclo et al. [9]. applied the more powerful BAHA (bone-anchored hearing aid) as a transcranial CROS device in patients with unilateral inner ear deafness. High patient satisfaction was reported as well as improvements in directional hearing. Later on, other studies also showed high patient satisfaction, but the improvements in directional hearing could not be replicated, which drew attention to the sound localization problems in patients with unilateral inner ear deafness [1012]. Good speech-in-noise results reflected the benefit of a BAHA CROS in lifting the head shadow while the compact design avoided some of the disadvantages of a conventional CROS. Therefore, the BAHA CROS is becoming more and more popular in patients with unilateral inner ear deafness [13].

Another option for patients with unilateral inner ear deafness was introduced in the late 1980s [14]. It comprised a high-power conventional air conduction hearing aid, with a relatively long ear mould that fit deeply into the patient's deaf ear and left the best ear unoccluded. When the amplified signals were loud enough they caused vibration of the bony walls of the ear canal and middle ear, which stimulated the normal ear by means of bone conduction through the skull. To achieve substantial gain and to overcome the interaural attenuation of the skull, very tight fitting is required, i.e. deep within the bony ear canal.
This application, therefore, forms an alternative transcranial CROS device that is fitted completely in the canal (CIC). Valente et al. [15] reported a success rate of $50 \%$ in their patients, which was high in comparison with their success rate of $10 \%$ with conventional CROS application. The improvement was ascribed to better sound quality: the harshness or tinny sound was gone. Hayes and Chen [16] also reported on the CIC device, but their study group only comprised three cases. As far as we know, little attention has been paid to this type of transcranial CROS method, since then [14, 17].

Faced with the increasing demands of communication skills in modern society, professionals are being urged more and more to recognize the detrimental effects of unilateral inner ear deafness. Currently, these patients can choose to learn various coping strategies, or they can give preference to receive unilateral amplification by means of a CROS hearing aid. Little has been published about the conventional CROS hearing aid or the CIC device. Although mostly poor results have been reported in the few available studies, some patients do benefit from these forms of CROS application.

Recently, many patients with unilateral sensorineural hearing loss become aware of the BAHA CROS option, not least due to its high profile on the internet [18]. Baguley et al. reviewed the published studies to emphasize the need for evidence base for the application of the BAHA CROS in these patients with SSD [18]. The reviewed studies found evidence of improved performance with the BAHA CROS [10, 11, 19]. However, Baguley et al. [18] criticized these studies on methodological aspects. All the studies, however, recommended careful selection of patients for the BAHA CROS as it was particularly advantageous in specific listening situations, but did not lead to objectively measurable improvements in directional hearing. Baguley et al., therefore, advised clinicians to proceed with caution and to await the outcome of a larger randomized trial [18, 20]. The largest series of patients in our clinic with unilateral sensorineural hearing loss who have been fitted with a BAHA CROS recently reached 56 [21]. These patients were selected on the basis of a preoperative trial with the BAHA CROS on a headband. On an average, the majority of these 56 patients are satisfied [21].

On the account of the criticism from Baguley, we performed a pilot study in which the three currently available CROS devices (conventional CROS, CIC and BAHA CROS) were assigned in random order to ten adult patients with unilateral sensorineural hearing loss for a test period of 8 weeks per device.

The evaluation comprised audiometric testing and patient outcome measurements on three hearing specific instruments to quantify subjective benefit. 


\section{Patients and methods}

\section{Patients}

At our outpatient clinic, we recruited ten adult patients with unilateral sensorineural hearing loss. These patients had not necessarily visited our outpatient clinic to obtain information about hearing aids. They were invited to participate in a prospective trial with three different methods of unilateral amplification: the conventional CROS (CROS), the CIC and the BAHA CROS on a headband (BAHA).

Each subject's unaided performance provided a baseline measurement to assess the effectiveness of the CROS devices. All the patients had normal hearing (PTA $<25 \mathrm{~dB}$ ) in the contralateral ear with a mean PTA of $12 \mathrm{dBHL}$. These thresholds meet the current conventional criteria for the SSD indication. Two patients had congenital unilateral hearing loss, the other eight patients had acquired unilateral hearing loss due to acoustic neuroma excision $(n=1)$, trauma $(n=3)$, meningitis $(n=2)$ and sudden deafness with cause unknown $(n=2)$. Average duration of deafness was 23 years (range $1-56$ ). Table 1 presents an overview of gender, age, etiology and duration of unilateral hearing loss.

\section{Methods}

Patients were offered a trial period with each of the three CROS devices for a period of 8 weeks in a random order. This duration is based on the literature on acclimatization by Gatehouse [22]. The conventional CROS test device consisted of a behind the ear hearing aid with a wire around the neck (Widex B2 with Widex CROS unit on the contralateral side). The Beltone deeply fitted device comprised a P60PP CIC hearing aid, which was tested after a deeply fitted patient-specific mould had been made. Fitting parameters of both devices were set according to the specifications of the manufacturer. The frequency response of the hearing aids was set to a maximally flat response. The gain of the CIC was set to the feedback limit. The effectiveness of the transcranial CIC is assessed by means of standard procedures. The BAHA CROS was worn on a steel headband with adequate coupling force, as a good relation (transcutaneous vs. percutaneous loss is around $10 \mathrm{~dB}$ ) is seen between the results with the headband and the BAHA on an implant $[23,24]$. Of course, the BAHA on a headband is the only option to test the device on a trial basis.

Patients were encouraged to use the devices on a daily basis. The CROS devices were fitted without providing any information about efficacy or comfort. At the end of the trial, the patients were asked whether they felt that the CROS amplification had been worthwhile and which of the three CROS devices (if any) took their preference.

Evaluations were made in four different conditions: unaided, with the conventional CROS, with the CIC hearing aid and with the BAHA CROS on a headband.

The data obtained in the unaided condition served as a baseline measurement to assess the effectiveness of the hearing aids. Afterwards, the patients were fitted with one of the three devices for a trial period of 8 weeks. In all four conditions, the audiometric evaluation consisted of sound localization measurements with a 9 -speaker array at $30^{\circ}$, for details see Bosman et al. [25]. From that experiment, we deleted the response to $0^{\circ}$ azimuth stimulation and determined simply whether the respond was on the side of the stimulation; this is referred to as the lateralization score. Speech perception was measured using short, everyday Dutch sentences [26]. Spectrally, shaped noise at a fixed level of $65 \mathrm{~dB}$ was presented in front of the listener (at azimuth $0^{\circ}$ ) and speech at $+90^{\circ}$ or at $-90^{\circ}$ azimuth and vice versa. Speech reception thresholds were measured with the "one up-one down" adaptive tracking procedure

Table 1 Patient characteristics: gender, age, etiology, duration of unilateral deafness and average pure tone average (PTA) in dBHL at the frequencies 500, 1,000 and 2,000 $\mathrm{Hz}$ in the normal ear

\begin{tabular}{|c|c|c|c|c|c|c|}
\hline Patient & Gender & Age (years) & Etiology & Duration (years) & PTA BC & PTA AC \\
\hline 1 & M & 31 & Congenital & 31 & 8.3 & 13.3 \\
\hline 2 & M & 51 & Trauma & 41 & 8.3 & 8.3 \\
\hline 3 & M & 64 & Acoustic neuroma & 10 & 0.0 & 3.3 \\
\hline 4 & $\mathrm{~F}$ & 56 & Congenital & 56 & 5.0 & 5.0 \\
\hline 5 & M & 45 & Trauma & 1 & 8.3 & 8.3 \\
\hline 6 & $\mathrm{~F}$ & 28 & Meningitis & 27 & 13.3 & 18.3 \\
\hline 7 & M & 47 & Trauma & 3 & 23.3 & 25.0 \\
\hline 8 & $\mathrm{~F}$ & 44 & Sudden deafness & 1 & 13.3 & 13.3 \\
\hline 9 & $\mathrm{~F}$ & 53 & Meningitis & 41 & 13.3 & 13.3 \\
\hline 10 & M & 43 & Sudden deafness & 18 & 8.0 & 8.0 \\
\hline
\end{tabular}

$A C$ air conduction, $B C$ bone conduction 
described by Plomp and Mimpen [26]. The same measurement setup as in our previous studies was chosen to avoid methodological differences.

\section{Patient outcome measures}

The baseline (unaided) and post-intervention measurements were conducted using two validated instruments in the Dutch language: the abbreviated profile of hearing aid benefit (APHAB) [27] and the speech, spatial and qualities (SSQ) of hearing profile [28]. The general SSD questionnaire was added to obtain more specific information. Patients were asked to fill out the post-intervention instruments at the end of each trial period after 8 weeks of experience with each device.

The abbreviated profile of hearing aid benefit (APHAB) [27] consists of 24 items in the following domains: ease of conversation (EC), listening under reverberant conditions $(\mathrm{RV})$, listening in background noise (BN) and aversiveness to loud sounds (AV). Higher scores reflect more problems.

The SSQ of hearing scale [28] measures benefit on the domains spatial hearing, speech perception and quality of sounds. As the spatial hearing domain is presumed to be of importance to binaural hearing, we only used the 16 items from the spatial hearing domain of the questionnaire (e.g. locate speaker round a table, locate dog barking, judge distance of a vehicle, etc.). High scores reflect good performance. This instrument is thought to be sensitive to auditory disabilities associated with SSD, especially.

The 12-item SSD questionnaire [29] was also administered to obtain data on the use, satisfaction, estimation of hearing aid benefit in different listening situations in comparison with the unaided situation, esthetics and handling of the CROS devices. This questionnaire has been used in previous studies on unilateral inner ear deafness [29].

\section{Results}

General

One patient did not participate in all unaided audiometric measurements. Two patients could not be fitted with the completely in the CIC, due to an enlarged external meatus secondary to tumor excision. One of these two patients dropped out, resulting in both CIC and CROS measurements were lacking. Another patient tried all three hearing aids, but was unable to complete the audiometric testing. In addition, for this patient most of the questionnaires were too difficult to fill out adequately, even with assistance. All the patients filled out the patient outcome measures in nearly all the conditions. When data were missing, this is specified.

\section{Source localization}

Sound localization performance was essentially at chance level in all four conditions. Data on lateralization (left/ right) scores are shown in Table 2.

\section{Speech recognition}

Speech reception thresholds (SRTs) in noise in all four conditions are shown in Fig. 1. Better performance corresponds with lower signal-to-noise ratios. Data were collected with the noise in front, while the speech was presented on either the profoundly deaf side (PE) or the normal hearing side (BE). This condition was called lateral speech. In the unaided condition (see Fig. 1), the S/N ratio when the speech was presented on the deaf side with the noise in front was about $0.5 \mathrm{~dB}$. In the aided conditions, these $\mathrm{S} / \mathrm{N}$ ratios were better with the conventional CROS (reduced to about $-1.7 \mathrm{~dB}$ ), slightly poorer with the BAHA CROS $(0.4 \mathrm{~dB})$ and somewhat poorer with the CIC (about $0.7 \mathrm{~dB}$ ).

When the speech was presented on the normal side (best ear $=\mathrm{BE}$ ), the $\mathrm{S} / \mathrm{N}$ ratio in the unaided condition was $-4.3 \mathrm{~dB}$. With the conventional CROS, the CIC and the BAHA CROS, these $\mathrm{S} / \mathrm{N}$ ratios were $-2.7,-4.6$ and $-2.0 \mathrm{~dB}$, respectively.

Outcome measures

Abbreviated profile of hearing aid benefit

Scores on the different domains of the APHAB are shown in Fig. 2. The lower the score, the better is the outcome.

Table 2 Average sound lateralization scores using 500 and 3,000 Hz stimuli in four conditions: unaided, conventional CROS (CROS), completely in the canal (CIC) and BAHA CROS (BAHA)

\begin{tabular}{lllllll}
\hline & $\begin{array}{l}\text { Chance level } \\
(\%)\end{array}$ & $\begin{array}{l}\text { Frequency } \\
(\mathrm{Hz})\end{array}$ & $\begin{array}{l}\text { Unaided (\%) } \\
(n=10)\end{array}$ & $\begin{array}{l}\text { CROS (\%) } \\
(n=8)\end{array}$ & $\begin{array}{l}\text { CIC (\%) } \\
(n=7)\end{array}$ \\
\hline Lateralization & 50 & 500 & 53.6 & 53.0 & 53.3 & 56.1 \\
& & 3,000 & 61.0 & 48.6 & 70.4 & 58.9 \\
\hline
\end{tabular}

Chance level for lateralization (50\%) is shown 

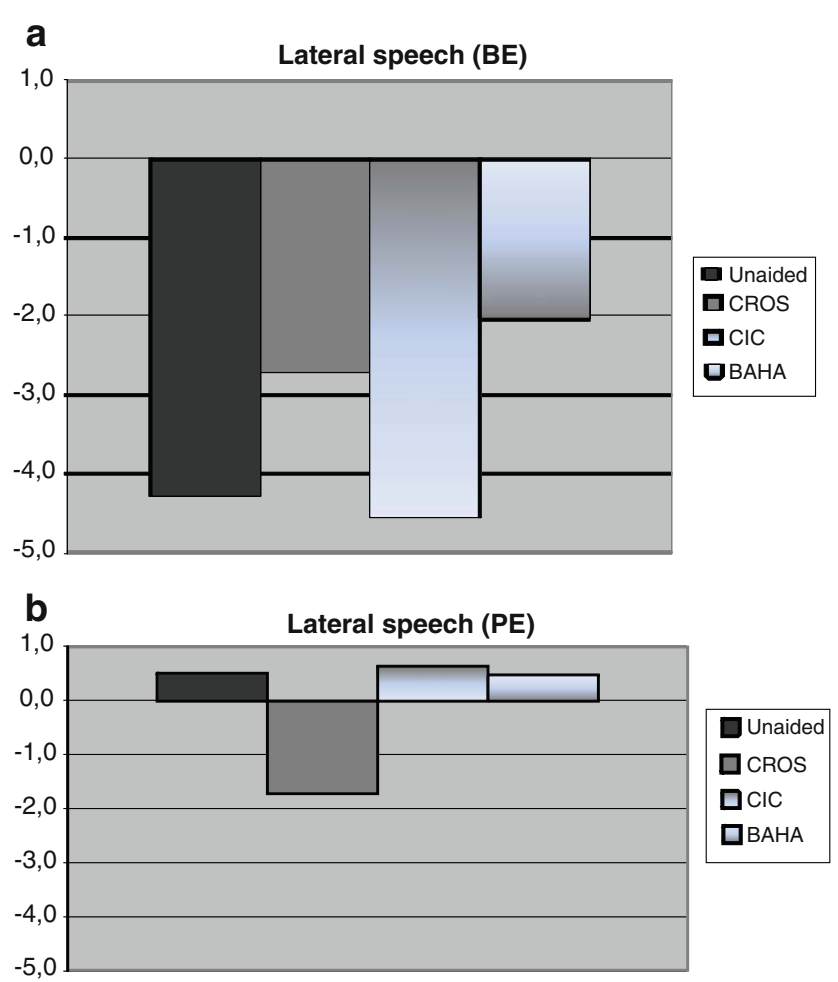

Fig. 1 a, b S/N for a speech intelligibility of $50 \%$ with everyday Dutch sentences: with noise presented in front and speech on either the poor ear (PE) or the best ear (BE). 'Lateral speech' in four conditions: unaided $(n=9)$, conventional CROS (CROS, $n=8)$, completely in the canal (CIC, $n=7$ ) and BAHA CROS (BAHA, $n=9)$

The conventional CROS showed improved scores on the domains EC (22), BN (61) and RV (38). All three hearing aids showed deterioration on the domain AV. The least deterioration was seen with the conventional CROS (40).

The CIC showed an improved score on the domain EC (26), but poorer scores on the other domains BN (73), RV (53) and AV (40).

The BAHA CROS showed the largest improvement on the domain EC (18) and less pronounced improvement on the domain BN (54). The scores were poorer on the domains RV (46) and AV (42). Overall, the conventional CROS had the best scores on the APHAB domains.

\section{Speech, spatial and qualities}

The results are presented in Table 3, the higher scores, the better is the outcome. The mean score on the spatial domain in the unaided condition $(n=10)$ was 3.7 on a scale from 0 to 10 . In the aided conditions with the CROS $(n=8)$, CIC $(n=8)$ and BAHA $(n=9)$ the mean scores were better: $1.3,0.3$ and 1.1 , respectively.

\section{Speech, spatial and qualities}

Most of the patients used each of the CROS devices more than $8 \mathrm{~h}$ a day (CIC), or 4-8 $\mathrm{h}$ a day (CROS, BAHA), 67 days a week. With the CIC, the best subjective scores were found on the domains wearing comfort, easy to use, rustle, whistle and failure. Lower scores were assigned to the conventional CROS and the BAHA CROS. In contrast, most of the patients $(n=6)$ said that the BAHA CROS was beneficial to hearing, whereas the CIC was of no benefit $(n=6)$ (see Fig. 3). The average score on several quality of sound items was best with the CROS (7 on a
Fig. 2 Mean scores of the 10 patients on the APHAB in the domains ease of communication (EC), background noise (BN), reverberation $(\mathrm{RV})$ and aversiveness of sound (AV) of the APHAB in four different conditions: unaided $(n=10)$, conventional CROS (CROS, $n=8)$, completely in the canal $(\mathrm{CIC}, n=8)$ and BAHA CROS (BAHA, $n=9$ )

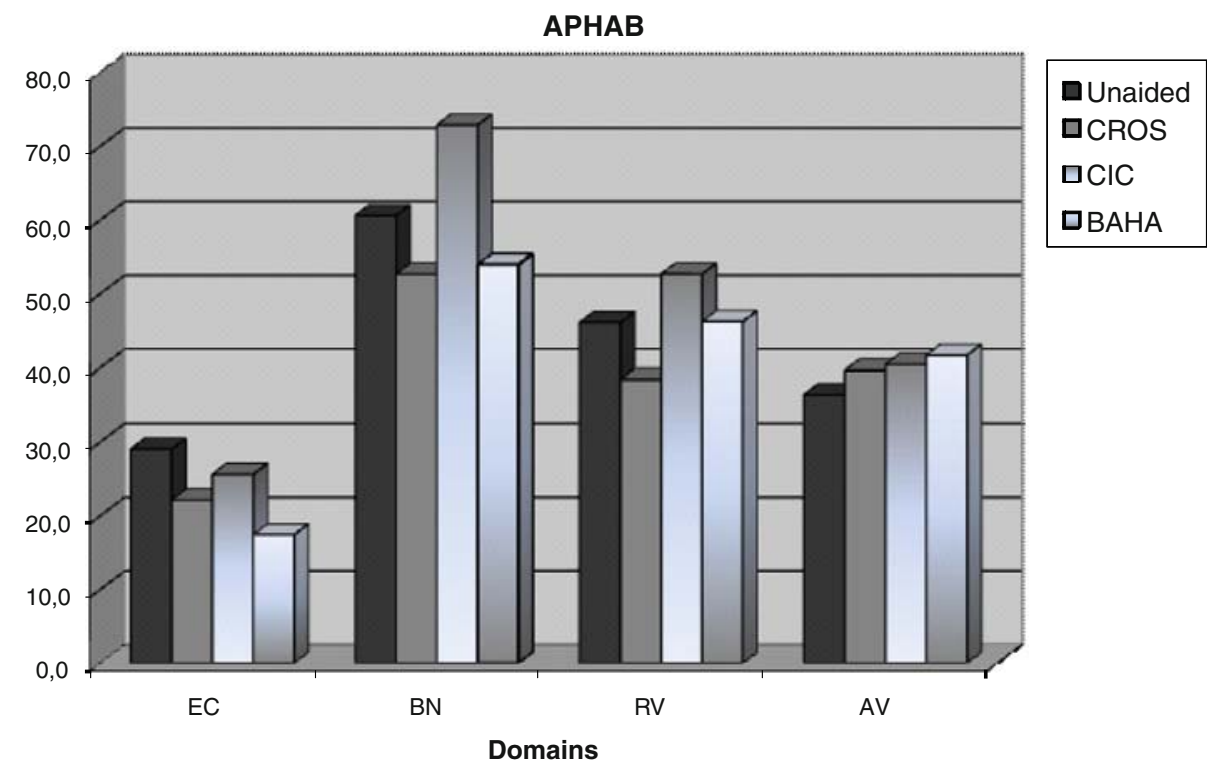


Table 3 Mean scores of the ten patients on the spatial domain of the SSQ in the four conditions: unaided, conventional CROS (CROS), completely in the canal (CIC) and BAHA CROS (BAHA)

\begin{tabular}{llc}
\hline SSQ & Mean & Difference \\
\hline Unaided & $3.7(1.5)$ & $\cdot$ \\
CROS & $5.0(1.8)$ & +1.3 \\
CIC & $4.0(1.4)$ & +0.3 \\
BAHA & $4.8(2.5)$ & +1.1 \\
\hline
\end{tabular}

scale from 0 to 10 ), slightly lower with the BAHA (6.8) and very poor with the CIC (3.7).

Following the completion of the trial, the patients were asked whether they wished to apply for one of the three CROS devices. Six patients declined, three patients opted for a BAHA CROS (nos. 6, 8 and 10, see Table 1) and one patient chose the conventional CROS with FM link (no. 3, see Table 1). The first two BAHA CROS patients have been implanted and they are satisfied with its performance.
The third patient is awaiting surgery. None of the patients who participated in our trial chose the CIC.

\section{Discussion}

In this pilot study, three different unilateral CROS amplification options were tested by patients with unilateral inner ear deafness. The patients' experience in this pilot was intended to act as the beginning of a large evidencebased study for patients with difficulties in daily life who are looking for a solution. The BAHA CROS has been found to alleviate the head shadow effect $[10,11,19,30]$. The conventional CROS is known to have its own merits, but also disadvantages [31]. The completely in the CIC, an alternative transcranial CROS application, has received only sparse attention in the literature.

The BAHA CROS is the only system that requires osseointegration and, therefore, surgery in preparation for
Fig. 3 a Single-sided deafness (SSD) questionnaire results, with the CROS $(n=8)$, CIC $(n=7)$ and the BAHA $(n=9)$. Subjective benefit with each hearing aid was scored in five domains (wearing comfort, easy to use, rustle, whistle and failure) on a scale from 0 to 10 . b Number of patients who indicated benefit with one of the hearing aids is indicated on the $y$ axis. The percentage is indicated in the figure itself
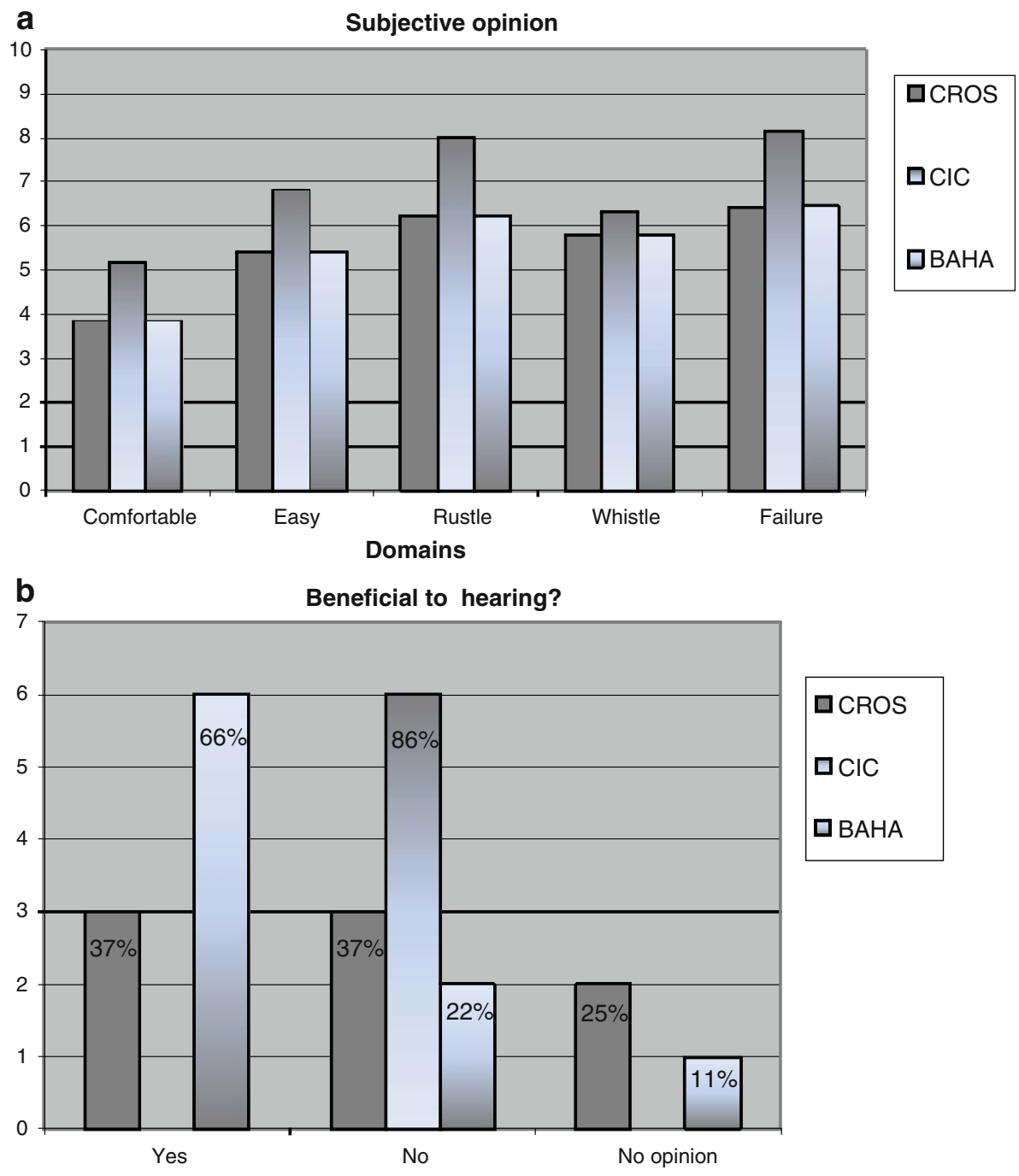
fitting. However, it can also be tested preoperatively by means of a transcutaneous setup in which the BAHA is connected to a special plastic disc held in place by a steel spring headband. If the patients are satisfied with this transcutaneous application, they might experience even more benefit from the ultimate bone conduction system after implant surgery.

This pilot study has investigated the experience of ten patients with three different CROS devices which they tried out in a random order for equal periods of time. For logistic reasons, the number was at first set on ten patients. To provide true evidence-based results, a larger study is required for these treatments in patients with unilateral sensorineural hearing loss [32, 33].

The directional hearing measurements obtained in this study confirmed our previous findings in patients with unilateral deafness: the results were essentially at chance level, irrespective of which device the patients were using $[10,29]$. In our setup, the patients were instructed to keep their head facing to the front. The scores at $3,000 \mathrm{~Hz}$ were somewhat poorer with the CROS and BAHA than in the unaided condition, but there was a small improvement with the CIC. However, none of the scores were statistically significant. Despite the shortcomings (e.g. low number of participants with missing data), this was the first random-controlled trial that not only evaluated the conventional CROS and the BAHA CROS, but also the CIC. In our opinion, the trend in our results is of value. The poor sound localization results are in agreement with the previous studies with larger numbers; binaural hearing cannot be achieved [21]. A remark can be made concerning the setup of our directional hearing measurements; they do not resemble real life as patients were instructed to keep their head still and the sounds used are not everyday sounds. This setup was chosen, however, to make the pilot group comparable to our previous data. Further research with more 'life-like' tests is still under construction in our clinic.

A major drawback of this pilot study is the small number of patients. It was remarkable that the CIC produced favorable SRT results, but none of the patients ultimately chose the CIC. This indicates once more that the decision of a patient to wear a certain hearing aid is not just audiological, but extends beyond the boundaries of objective results. Our ultimate goal is to achieve an instrument that will be able to capture these "non-objective" data and turn them into a valuable tool for preoperative selection.

Patient outcome measures are essential in this evaluation of patient benefit, still the one and only perfect instrument does not exist yet. For the moment, we choose those instruments that were previously used in similar studies for subjective evaluation. We used three different instruments: the APHAB, the SSQ and the SSD questionnaire.
In general, the APHAB showed the poorest scores with the CIC, the best scores with the BAHA and intermediate scores with the conventional CROS.

To gather subjective information about auditory localization, the spatial part of the SSQ questionnaire was used. Some benefit in directional hearing was found with each of the three devices. The BAHA and the conventional CROS showed the most benefit (overall improvement of 1.1 and 1.3, respectively). In comparison, Noble and Gatehouse obtained a mean unaided score of 4.8 from 50 patients with asymmetrical sensorineural hearing loss [28].

In addition, the general SSD questionnaire was used. Remarkably, the CIC had the best scores on the SSD items wearing comfort, easy to use, rustle, whistle and failure. The average score on several quality of sound items was best with the conventional CROS (7.0), slightly poorer with the BAHA (6.8) and very poor with the CIC (3.7).

At the end of this trial, six out of the ten patients did not choose any of the unilateral amplification methods they tested in this study. The one patient who chose the conventional CROS is using an FM link instead of a wire around the neck. The two implanted BAHA CROS patients are satisfied with the performance of the device; the third patient who chose the BAHA CROS is awaiting surgery.

This pilot study was performed to evaluate several items. First of all, if the decision for a specific CROS hearing aid was dependent on the order in which they were trialed? This was not the case. Second, if we should offer each of our SSD patients the personally designed CIC? None of the patients chose the CIC, presumably based on the subjectively experienced poor quality of sound. To answer this question correctly, we should have verified to what degree the amplified speech was audible to the better hearing ear. However, the SRT results improve indicating effective amplification. We choose in our clinic to not regularly test the CIC in our SSD patients, as none of the pilot study patients chose the CIC. Third, what is the success rate of BAHA CROS in SSD patients, not necessarily experiencing hearing problems? In literature, it is reported that $25 \%$ of the patients, who underwent acoustic neuroma surgery, apply for implantation of the BAHA system after trial on a headband [34]. Our percentage of $30 \%$ (3 out of 10 patients) who chose the BAHA is in accordance with these reports. Fourth, what are the selection criteria to preoperatively select a suitable BAHA CROS patient? This study again shows that the trial of the BAHA headband is predictive and useful. More research is needed, however, to develop an instrument to identify the patient that will benefit from a CROS hearing aid. It can be suggested that, as Harford and Dodds [1] already found, the degree of success depends on the motivation of the patient and the listening demands imposed by their lifestyle and working environment. This pilot study showed again that these demands are not measured within the currently used instruments (APHAB and 
SSQ), therefore, our clinic is intending in establishing an instrument capturing these demands.

\section{Conclusion}

In conclusion, most of the patients experienced some degree of benefit with each of the three CROS devices, but it was most of the time not large enough to outweigh the disadvantages. Preference for one of the three hearing aids was independent of the order in which they were trialed. We recommend that all patients with unilateral inner ear deafness, not only those applying for a hearing aid, but also those without hearing problems, should be offered a trial with at least the BAHA CROS on a headband. It would be worthwhile to formulate selection criteria to help establish if a CROS hearing aid will be beneficial and preferably also which CROS hearing aid will suit a specific patient.

Open Access This article is distributed under the terms of the Creative Commons Attribution Noncommercial License which permits any noncommercial use, distribution, and reproduction in any medium, provided the original author(s) and source are credited.

\section{References}

1. Harford E, Dodds E (1966) The clinical application of CROS: a hearing aid for unilateral deafness. Arch Otolaryngol 83(5):455-464

2. Lotterman SH, Kasten RN (1971) Examination of the CROS type hearing aid. J Speech Hear Res 14(2):416-420

3. Markides A (1979) The CROS hearing aid system. Br J Audiol 13(2):63-72

4. Harford E, Barry J (1965) A rehabilitative approach to the problem of unilateral hearing impairment: contralateral routing of signals (CROS). J Speech Hear Disord 30:121-138

5. Valente M (1995) Fitting options for unilateral hearing. Hear J 48(10):45-48

6. Fowler EP (1960) Bilateral hearing aids for monaural total deafness: a suggestion for better hearing. Arch Otolaryngol 72:41-42

7. Welling DB, Glasscock ME III, Woods CI, Sheffey RC (1991) Unilateral sensorineural hearing loss rehabilitation. Otolaryngol Head Neck Surg 105(6):771-776

8. Snik AF, Dreschler WA, Tange RA, Cremers CW (1998) Shortand long-term results with implantable transcutaneous and percutaneous bone-conduction devices. Arch Otolaryngol Head Neck Surg 124(3):265-268

9. Vaneecloo FM, Hanson JN, Laroche C, Vincent C, Dehaussy J (2000) [Prosthetic rehabilitation of unilateral anakusis. Study with stereoaudiometry]. Ann Otolaryngol Chir Cervicofac 117(6):410-417

10. Hol MKS, Bosman AJ, Snik AFM, Mylanus EAM, Cremers CWRJ (2004) Bone anchored hearing aid in unilateral inner ear deafness: a study of 20 patients. Audiol Neurootol 9(5):274-281

11. Niparko JK, Cox KM, Lustig LR (2003) Comparison of the bone anchored hearing aid implantable hearing device with contralateral routing of offside signal amplification in the rehabilitation of unilateral deafness. Otol Neurotol 24(1):73-78

12. Wazen JJ, Ghossaini SN, Spitzer JB, Kuller M (2005) Localization by unilateral BAHA users. Otolaryngol Head Neck Surg 132(6):928-932
13. Snik AF, Mylanus EA, Proops DW et al (2005) Consensus statements on the BAHA system: where do we stand at present? Ann Otol Rhinol Laryngol Suppl 195:2-12

14. Sullivan RF (1988) Transcranial ITE CROS. Hear Instrum 39:54

15. Valente M, Potts L, Valente M, Goebel J (1995) Wireless CROS versus transcranial CROS for unilateral hearing loss. Am J Audiol 4(1):52-59

16. Hayes DE, Chen JM (1998) Bone-conduction amplification with completely-in-the-canal hearing aids. J Am Acad Audiol 9(1):5966

17. Fagelson MA, Noe CM, Murnane OD, Blevins JS (2003) Predicted gain and functional gain with transcranial routing of signal completely-in-the-canal hearing aids. Am J Audiol 12(2):84-90

18. Baguley DM, Bird J, Humphriss RL, Prevost AT (2006) The evidence base for the application of contralateral bone anchored hearing aids in acquired unilateral sensorineural hearing loss in adults. Clin Otolaryngol 31(1):6-14

19. Wazen JJ, Spitzer JB, Ghossaini SN et al (2003) Transcranial contralateral cochlear stimulation in unilateral deafness. Otolaryngol Head Neck Surg 129(3):248-254

20. Baguley DM, Plydoropulou V, Prevost AT (2009) Bone anchored hearing aids for single-sided deafness. Clin Otolaryngol 34(2):176-177

21. Kunst SJW (2008) Bone-anchored hearing aid in patients with acquired and congenital unilateral inner ear deafness (BAHA CROS): clinical evaluation of 56 cases. Thesis, pp 89-106

22. Gatehouse S (1993) Role of perceptual acclimatization in the selection of frequency responses for hearing aids. J Am Acad Audiol 4(5):296-306

23. Carlsson PU, Hakansson BE (1997) The bone-anchored hearing aid: reference quantities and functional gain. Ear Hear 18(1):34-41

24. Håkansson BE, Carlsson PU, Tjellström A, Liden G (1994) The bone-anchored hearing aid: principal design and audiometric results. Ear Nose Throat J 73(9):670-675

25. Bosman AJ, Snik AFM, van der Pouw CTM, Mylanus EAM, Cremers CWRJ (2001) Audiometric evaluation of bilaterally fitted bone-anchored hearing aids. Audiology 40(3):158-167

26. Plomp R, Mimpen AM (1979) Improving the reliability of testing the speech reception threshold for sentences. Audiology 18(1):43-52

27. Cox RM, Alexander GC (1995) The abbreviated profile of hearing aid benefit. Ear Hear 16(2):176-186

28. Gatehouse S, Noble W (2004) The speech, spatial and qualities of hearing scale (SSQ). Int J Audiol 43(2):85-99

29. Hol MKS, Bosman AJ, Snik AFM, Mylanus EAM, Cremers CWRJ (2005) Bone-anchored hearing aids in unilateral inner ear deafness: an evaluation of audiometric and patient outcome measurements. Otol Neurotol 26(5):999-1006

30. Vaneecloo FM, Ruzza I, Hanson JN et al (2001) The monaural pseudo-stereophonic hearing aid (BAHA) in unilateral total deafness: a study of 29 patients. Rev Laryngol Otol Rhinol (Bord) 122(5):343-350

31. Valente M, Valente M, Meister M, Macauley K, Vass W (1994) Selecting and verifying hearing aid fittings for unilateral hearing loss. In: Valente M (ed) Strategies for selecting and verifying hearing aid fittings. Thieme, Stuttgart, pp 228-248

32. Gabbard SA, Schryer J (2003) Early amplification options. Ment Retard Dev Disabil Res Rev 9:236-242

33. Snik AFM, Mylanus EAM, Cremers CWRJ (1995) The boneanchored hearing aid compared with conventional hearing aids: audiologic results and the patients' opinions. Otolaryngol Clin North Am 28(1):73-83

34. Andersen HT, Schroder SA, Bonding P (2006) Unilateral deafness after acoustic neuroma surgery: subjective hearing handicap and the effect of the bone-anchored hearing aid. Otol Neurotol 27(6):809-814 